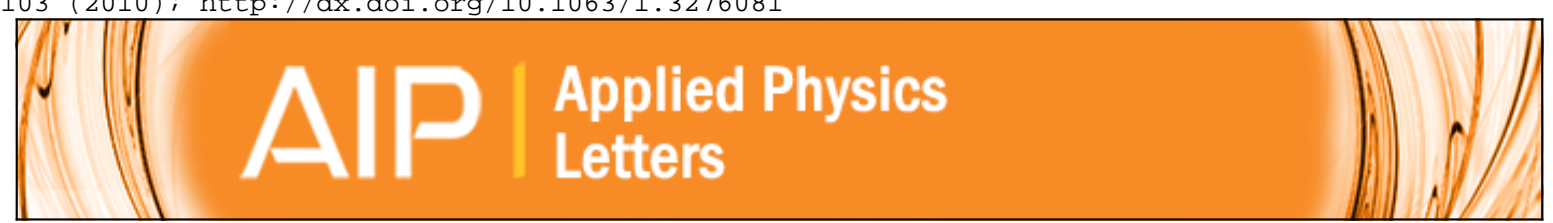

Development of a nonlinear nanoprobe for interferometric autocorrelation based characterization of ultrashort optical pulses

Haifeng Li, Yaoshun Jia, Qian Xu, Kebin Shi, Jian Wu, Peter C. Eklund, Yong Xu, and Zhiwen Liu

Citation: Applied Physics Letters 96, 021103 (2010); doi: 10.1063/1.3276081

View online: http://dx.doi.org/10.1063/1.3276081

View Table of Contents: http://scitation.aip.org/content/aip/journal/apl/96/2?ver=pdfcov

Published by the AIP Publishing

Over 700 papers \&
presentations on

multiphysics simulation nww now

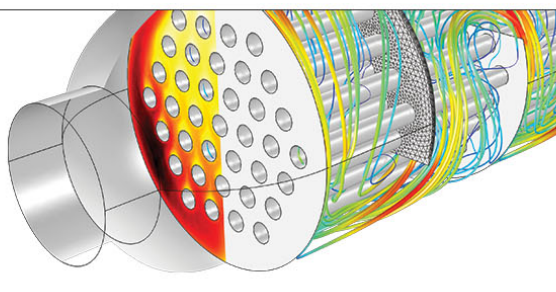




\title{
Development of a nonlinear nanoprobe for interferometric autocorrelation based characterization of ultrashort optical pulses
}

\author{
Haifeng Li, ${ }^{1}$ Yaoshun Jia, ${ }^{2}$ Qian Xu, ${ }^{1}$ Kebin Shi, ${ }^{1}$ Jian Wu, ${ }^{3}$ Peter C. Eklund, ${ }^{3,4}$ Yong Xu, ${ }^{2, a)}$ \\ and Zhiwen Liu, ${ }^{1, b)}$ \\ ${ }^{1}$ Department of Electrical Engineering, The Pennsylvania State University, University Park, \\ Pennsylvania 16802, USA \\ ${ }^{2}$ Department of Electrical and Computer Engineering, Virginia Tech, Blacksburg, Virginia 24061, USA \\ ${ }^{3}$ Departments of Physics, The Pennsylvania State University, University Park, Pennsylvania 16802, USA \\ ${ }^{4}$ Department of Materials Science and Engineering, The Pennsylvania State University, \\ University Park, Pennsylvania 16802, USA
}

(Received 7 August 2009; accepted 7 November 2009; published online 11 January 2010)

\begin{abstract}
Near-field scanning can achieve nanoscale resolution while ultrashort pulse diagnostic tools can characterize femtosecond pulses. Yet currently it is still challenging to nonperturbatively characterize the near field of an ultrashort optical pulse with nanofemtoscale spatiotemporal resolution. To address this challenge, we propose to develop a nonlinear nanoprobe composed of a silica fiber taper, a nanowire, and nonlinear fluorescent spheres. Using such a nanoprobe, we also report proof-of-principle characterization of femtosecond optical pulse through interferometric autocorrelation measurement. (C) 2010 American Institute of Physics. [doi:10.1063/1.3276081]
\end{abstract}

By going toward an ever smaller length scale and an ever faster time scale, nanotechnology and ultrafast technology have led to numerous important discoveries such as carbon nanotubes ${ }^{1}$ and the optical frequency comb, ${ }^{2}$ and together have created new scientific frontiers such as biophotonics and nanophotonics. However, there still exists a fundamental knowledge gap at the intersection of nano and ultrafast technology. More specifically, on one hand, we can utilize near-field scanning optical microscopy (NSOM) to achieve near field imaging with nanoscale spatial resolution, and on the other hand, we can apply ultrashort pulse measurement techniques to characterize femtosecond laser pulses. Yet, currently it is still difficult to perform nonperturbative measurement of an ultrafast optical near field with nanofemtoscale spatiotemporal resolutions, which is important for many applications, such as studying the light-matter interaction at the nanoscale level (e.g., nanoscale control of the quantum dynamics), ${ }^{3}$ investigating plasmon dynamics in complex nanostructures, ${ }^{4,5}$ as well as understanding spatiotemporal evolution of ultrashort pulses in nanoscale structures and waveguides.

Current methods for ultrashort pulse characterization ${ }^{6}$ include autocorrelation, frequency resolved optical gating (FROG), and spectral phase interferometry for direct electric field reconstruction among others. It is often implicitly assumed that the spatial propagation and the temporal evolution of the ultrashort pulses are largely independent. Spatiotemporal characterization has been previously investigated to study spatiotemporal pulse distortions. ${ }^{7,8}$ With the continuing expansion of the application of ultrafast lasers (e.g., nonlinear microscopy), it also becomes important to characterize ultrashort pulses near the focal point of a lens, where the spatial and temporal evolution of the optical pulses is usually coupled together and can be highly complex. For instance, an interferometric method named SEA TADPOLE was reported, ${ }^{9}$ in which an optical fiber is utilized to sample the ultrafast

\footnotetext{
${ }^{a}$ Electronic mail: yong@vt.edu. Tel.: 540-231-2464.

${ }^{b)}$ Electronic mail: zliu@psu.edu. Tel.: 814-865-2362.
}

optical field at different locations near the focal point of a lens. It has been shown that this method can achieve submicron spatial resolution by using NSOM fiber probes. ${ }^{10}$ Earlier efforts to characterize ultrashort pulses near the foci also include interferometric autocorrelation ${ }^{11,12}$ and collinear second harmonic FROG ${ }^{13}$ based methods. Recently, a nanoFROG technique was developed in which individual second harmonic nanocrystals were dispersed on a substrate and used as a probe to characterize ultrashort pulse profile through FROG. ${ }^{14}$ This approach represents significant progress toward nanoscale spatial resolution. However, the requirement of substrate attachment may limit the applicability of the probe to characterizing the near fields of nanostructures possessing complex three-dimensional geometries. In this letter, we propose to develop a nonlinear nanoprobe for nanofemtoscale characterization. The proposed nanoprobe consists of a silica fiber taper, a $\mathrm{ZnS}$ nanowire attached to the tip of the taper, and nonlinear particles (i.e., two-photon fluorescent micro or nanospheres) attached to the nanowire. As a result, the proposed nonlinear nanoprobe can also be integrated into current NSOM and atomic force microscope technologies. We demonstrate proof-of-principle femtosecond pulse characterization by interferometric autocorrelation measurement using the nonlinear nanoprobes.

The fabrication of the proposed nonlinear nanoprobe is carried out in three steps. The first step is fiber taper fabrication. We used a fiber fusion splicer (Ericsson FSU-975) to fabricate fiber tapers from regular silica fibers by programming the fusion current and time. The diameter of the obtained taper tip is typically around $1-2 \mu \mathrm{m}$. Next, a single $\mathrm{ZnS}$ nanowire is attached to the fiber taper. The $\mathrm{ZnS}$ nanowires were synthesized by pulsed laser vaporization. ${ }^{15,16} \mathrm{~A}$ target of well-mixed $\mathrm{ZnS}$ (Alfa Aesar) and 10 at. \% Au powder (Alfa Aesar) was placed in a double-quartz tube system centered in a tube furnace operated at $950{ }^{\circ} \mathrm{C}$. A carrier gas of $100 \mathrm{SCCM} \mathrm{Ar} / 5 \% \mathrm{H}_{2}$ was introduced between the inner and outer quartz tubes, allowing the gas to be preheated before entering the reaction zone $\left(\mathrm{Ar} / \mathrm{H}_{2}\right.$ absolute pressure was 225 Torr). The nanowires were found to grow via the vaporliquid-solid mechanism. ${ }^{17}$ The typical diameter of the $\mathrm{ZnS}$ 

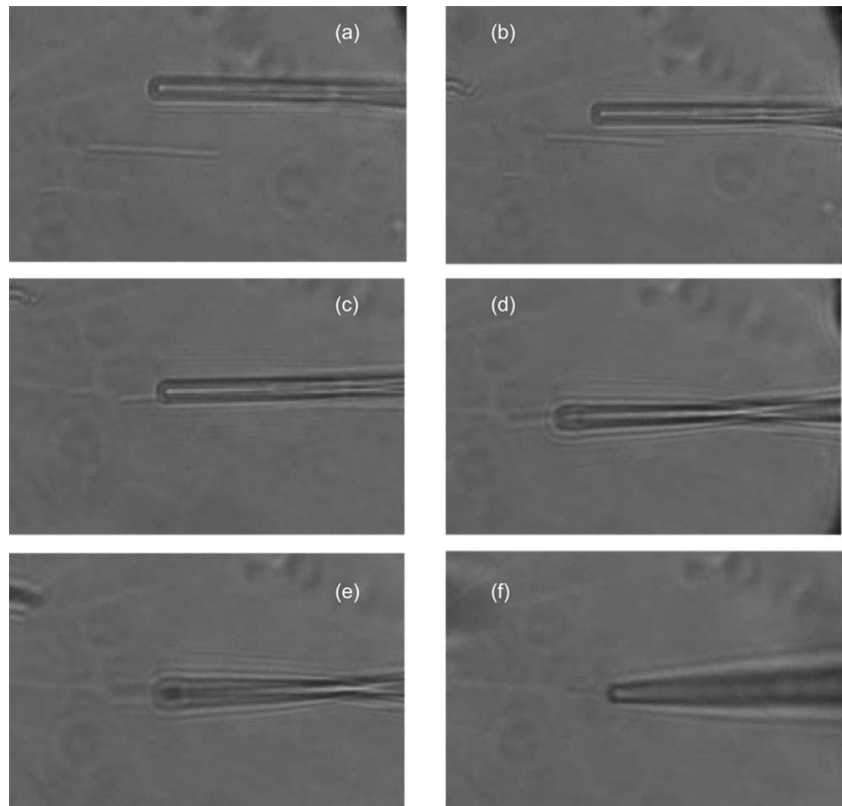

FIG. 1. A sequence of images showing the process of attaching a single $\mathrm{ZnS}$ nanowire to a fiber taper. [(a) and (b)] a fiber taper with a small amount of optical adhesive already applied to it was moved toward a $\mathrm{ZnS}$ nanowire; [(c)-(f)] the $\mathrm{ZnS}$ nanowire was attached to the fiber taper and lifted off the substrate as the taper was translated.

nanowires was found using SEM to be $100-200 \mathrm{~nm}$ and the lengths were typically in the range $\sim 20-30 \mu \mathrm{m}$. The $\mathrm{ZnS}$ nanowires were first dispersed on a glass substrate and then placed under a home-modified microscope system. A single $\mathrm{ZnS}$ nanowire was selected and attached to the fiber taper by using UV curable optical adhesive (Norland Optical Adhesive 60). Figure 1 shows a sequence of images illustrating this process. A ZnS nanowire can be seen in Fig. 1(a). A fiber taper (already with a small amount of optical adhesive applied to it) was moved toward the nanowire and attached to it as shown in Fig. 1(b)-1(f). Ultraviolet light exposure was then followed to cure the adhesive. Finally, nonlinear particles were attached to the nanowire. We fabricated two types of nanoprobes by attaching fluorescent micro and nanospheres (commercially available from Duke Scientific Corp.). The first type (type I) consists of a single fluorescent microsphere with a $1 \mu \mathrm{m}$ nominal diameter attached to the $\mathrm{ZnS}$ nanowire. The procedure is similar to that of attaching a nanowire to a taper except without the UV curing step. The second type (type II) of nanoprobe has multiple fluorescent nanospheres ( $\sim 100 \mathrm{~nm}$ in diameter) attached near the end of the $\mathrm{ZnS}$ nanowire. This was accomplished by first dispersing some fluorescent nanospheres on a separate fiber taper and then gently rubbing the nanowire on the nanospheres. The field emission scanning electron microscope images of two nanoprobes [type I-Fig. 2(a); and type II-Figs. 2(b) and 2(c)] are shown in Figs. 2(a) and 2(b). Figure 2(c) is a zoomed-in view of the tip of the type II nanoprobe shown in Fig. 2(b).

Two-photon excited fluorescence can be observed from the micro or nanospheres when excited by ultrashort pulses from a Ti:Sapphire laser (KMLabs, center wavelength $\sim 808 \mathrm{~nm}$, typical pulse width $\sim 60 \mathrm{fs}$, and typical average output power $\sim 500 \mathrm{~mW}$ ). To verify that the observed twophoton fluorescence was from the attached fluorescent spheres, we measured two-photon fluorescence spectra from

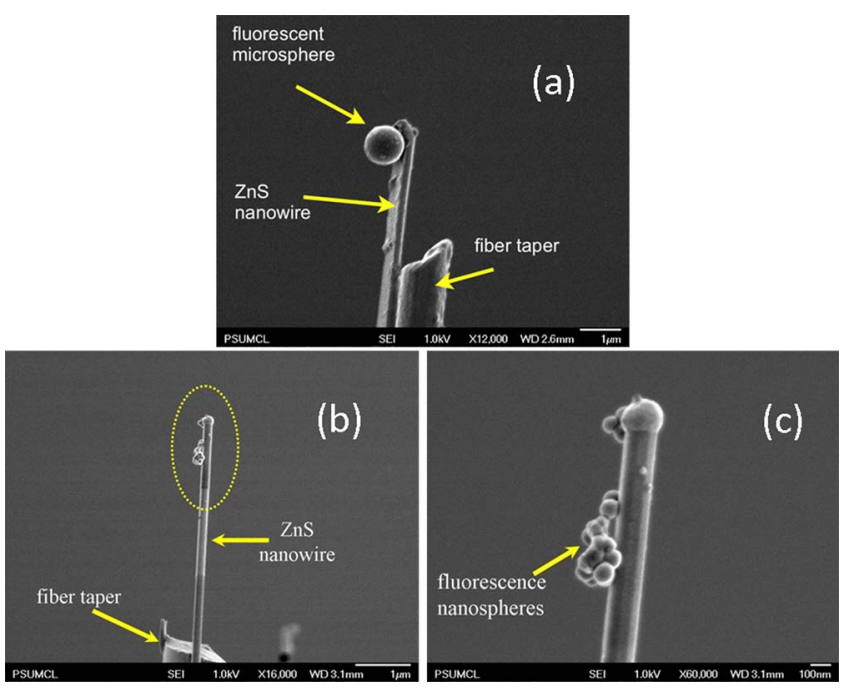

FIG. 2. (Color online) [(a) and (b)] field emission scanning electron microscope images of a nanoprobe attached with a single fluorescent microsphere $(\sim 1 \mu \mathrm{m}$ diameter) and a nanoprobe attached with multiple fluorescent nanospheres $(\sim 100 \mathrm{~nm}$ diameter), respectively; (c) zoomed-in view of the tip of the two photon nanoprobe shown in (b).

nanoprobes and spectra from $\mathrm{ZnS}$ nanowires only. The generated fluorescence signal was separated by filters and detected by a spectrograph with a liquid nitrogen cooled charge-coupled device camera (Princeton Acton, SP2500i). The results from type I and II nanoprobes are shown in Figs. 3(a) and 3(b), respectively. Within the sensitivity of our detection system, possible background contributions from the nanowire, the optical adhesive, and the fiber taper were not observed. Strong two-photon fluorescence signal from the fluorescent micro and nanospheres in the wavelength range from about 450 to $500 \mathrm{~nm}$ was observed as shown in Fig. 3 . The observed fluorescence signal significantly exceeds background noises, and is clearly due to the fluorescent spheres. It should be noted that if a single nanoparticle can be attached, nanometer scale spatial resolution in all three dimensions can be achieved and is limited by the size of the nanoparticle.

As a preliminary demonstration, we used the nanoprobes to characterize the pulse width of femtosecond laser pulses near the focal point of an objective lens. The schematic diagram of our experimental setup is shown in Fig. 4(a). Femtosecond beam from the Ti:Sapphire laser first passed through a long-pass filter, then entered a Michelson interferometer to produce two copies of the incoming pulses. Their relative time delay can be controlled by translating a mirror
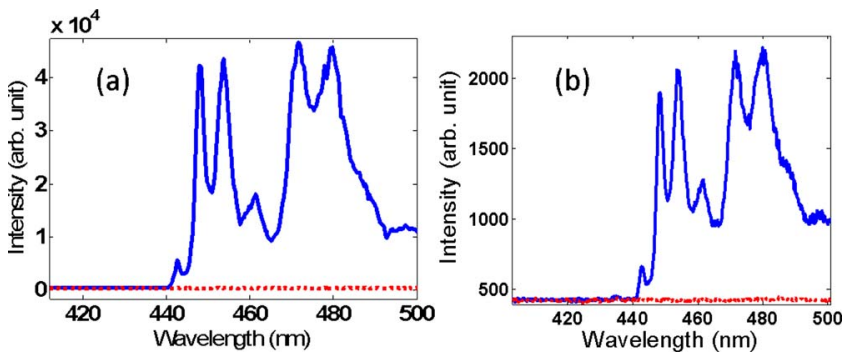

FIG. 3. (Color online) (a) two-photon fluorescence spectrum of a type I nanoprobe with a single $1 \mu \mathrm{m}$ sphere (solid blue curve) and spectrum of $\mathrm{ZnS}$ nanowire only (dotted red curve), (b) two-photon fluorescence spectrum of a type II nanoprobe with multiple fluorescent nanospheres $(\sim 100 \mathrm{~nm}$ diameter) (solid blue curve) and spectrum of $\mathrm{ZnS}$ nanowire only (dotted red curve) 


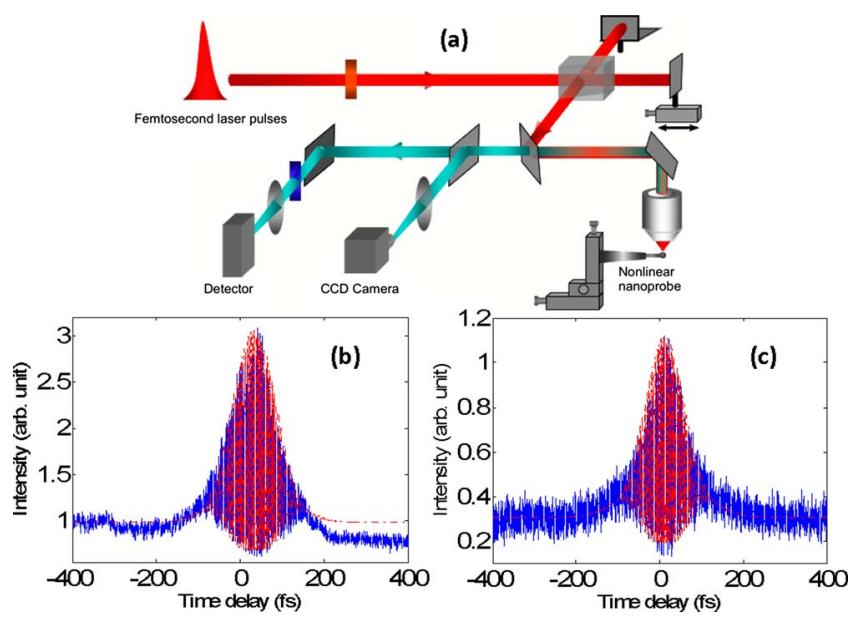

FIG. 4. (Color online) (a) Schematic diagram of the experimental system. (b) Measured interferometric autocorrelation trace by using a type I nanoprobe (blue) and theoretical curve (properly scaled and shifted) calculated by assuming a Gaussian pulse profile with a pulse width of 120 fs (full width at half maximum) and a linear chirp parameter $\alpha$ of 1.9 (red dots). (c) Interferometric autocorrelation trace obtained by using a type II nanoprobe (blue) and theoretical curve (properly scaled and shifted) calculated by assuming a Gaussian profile with a pulse width of $127 \mathrm{fs}$ (full width at half maximum) and a linear chirp parameter $\alpha$ of 2.5 (red dots).

in one arm of the interferometer. The output beam from the interferometer was subsequently focused by an objective lens $(60 \times$, numerical aperture: 0.85$)$ and a nanoprobe was placed in the focal point of the objective. Two-photon excited fluorescence signal from the nanoprobe was then gathered by the same objective lens, passed through a dichroic filter and then a combination of short-pass filters, and detected by a photomultiplier tube (HAMAMATSU H7827-011). The detected signal was further amplified with a lock-in amplifier. A chopper (not shown) placed near the laser output was used in conjunction with the lock-in amplifier. Interferometric autocorrelation traces can then be obtained by measuring the two-photon fluorescence signal as the delay time is scanned. In order to reduce the scanning time and improve the stability of the measurement, the fluorescence signal was recorded as the scanning mirror was translated continuously at a constant speed of $0.8 \mu \mathrm{m} / \mathrm{s}$ rather than by an incremental step each time. ${ }^{18}$ The typical acquisition time is about $2 \mathrm{~min}$. We first used a type I nanoprobe. The average laser power measured before the beam splitter was about $2 \mathrm{mw}$. The measured interferometric autocorrelation curve is shown in Fig. 4(b) (blue curve). By assuming a linearly chirped Gaussian pulse profile $E(t)=\exp \left[-(1+i \alpha)\left(t / \tau_{\mathrm{G}}\right)^{2}\right]$ the pulse width retrieved from this interferometric autocorrelation trace is about $120 \mathrm{fs}$ (full width at half maximum) and a chirp parameter $\alpha$ around 1.9 is estimated. ${ }^{6}$ The theoretical curve (properly scaled and shifted) obtained by using these parameters is also shown for comparison (red dots). It should be noted that the baseline of the interferometric autocorrelation curve is not even on both sides. This is likely due to slight instability of the probe during the experiment, which may cause the background to change during the course of the measurement. Compared with the thin $\mathrm{ZnS}$ nanowire, the $1 \mu \mathrm{m}$ fluorescent sphere is rather large and is likely more susceptible to environmental perturbation. This problem can be alleviated in the type II nanoprobe. We performed measurement by using the type II nanoprobe shown in Fig. 2(b). The average laser power before the beam splitter was about
$10 \mathrm{mw}$ in this case. The measured interferometric autocorrelation trace is shown in Fig. 4(c) (blue curve). Compared to the response of the type I nanoprobe, the signal strength is reduced. The retrieved pulse width is about $127 \mathrm{fs}$ (full width at half maximum) and a chirp parameter $\alpha \sim 2.5$ is estimated. The calculated theoretical curve is also shown in Fig. 4(c) (red dots). We notice that there is some difference between the two retrieved pulse widths and chirping parameters. This is likely due to the noise in the measurement as well as to possible slight changes in the laser cavity between the two experiments. The pulse broadening and chirping is primarily caused by the dispersive propagation of the laser pulses in a relatively thick cube beam splitter used in the interferometer for this preliminary study. Other contributions to pulse broadening and chirping include that from the objective and the dichroic filter used in the setup.

In conclusion, we have proposed and fabricated a nonlinear nanoprobe for characterization of ultrashort optical pulses. Proof-of-principle interferometric autocorrelation measurements near the focal point of an objective through two-photon fluorescence from such nonlinear nanoprobes were demonstrated. Since the bulk of the nanoprobe is built from nanoparticles attached to a dielectric nanowire, we can minimize optical scattering and produce minimally perturbative measurements. Nanoscale spatial resolution can be potentially achieved through near field scanning of the nonlinear nanoprobe. We believe that our technique can be further improved to incorporate other types of nonlinear nanoparticles and can potentially lead to full nanofemto spatiotemporal characterization of complex ultrafast optical near fields, which can find important applications in ultrafast nanophotonics.

This work is supported by the National Science Foundation (Grant Nos. 0649866, 0547475, and 0644488). H.L and Y.J. contributed equally to this work.

${ }^{1}$ R. H. Baughman, A. A. Zakhidov, and W. A. de Heer, Science 297, 787 (2002).

${ }^{2}$ T. Udem, R. Holzwarth, and T. W. Hänsch, Nature (London) 416, 233 (2002).

${ }^{3}$ M. Durach, A. Rusina, M. I. Stockman, and K. Nelson, Nano Lett. 7, 3145 (2007).

${ }^{4}$ A. Anderson, G. Steinmeyer, and M. B. Raschke, CLEO, CTHK2, (2009).

${ }^{5}$ A. Kubo, K. Onda, H. Petek, Z. Sun, Y. S. Jung, and H. K. Kim, Nano Lett. 5, 1123 (2005).

${ }^{6}$ J.-C. Diels and W. Rudolph, Ultrashort Laser Pulse Phenomena, 2nd ed. (Academic, Boston, 2006).

${ }^{7}$ C. Dorrer, E. M. Kosik, and I. A. Walmsley, Appl. Phys. B: Lasers Opt. 74 [Suppl.], s209 (2002).

${ }^{8}$ S. Akturk, M. Kimmel, P. O'Shea, and R. Trebino, Opt. Express 11, 68 (2003).

${ }^{9}$ P. Bowlan, P. Gabolde, and R. Trebino, Opt. Express 15, 10219 (2007).

${ }^{10}$ P. Bowlan, U. Fuchs, R. Trebino, and U. D. Zeitner, Opt. Express 16, 13663 (2008).

${ }^{11}$ M. Muller, J. Squier, and G. J. Brakenhoff, Opt. Lett. 20, 1038 (1995).

${ }^{12}$ J. B. Guild, C. Xu, and W. W. Webb, Appl. Opt. 36, 397 (1997).

${ }^{13}$ I. Amat-Roldán, I. G. Cormack, P. Loza-Alvarez, and D. Artigas, Opt. Lett. 29, 2282 (2004)

${ }^{14}$ J. Extermann, L. Bonacina, F. Courvoisier, D. Kiselev, Y. Mugnier, R. L. Dantec, C. Galez, and J.-P. Wolf, Opt. Express 16, 10405 (2008).

${ }^{15}$ A. M. Morales and C. M. Lieber, Science 279, 208 (1998).

${ }^{16}$ Q. Xiong, G. Chen, G. D. Acord, X. Liu, J. J. Zengel, H. R. Gutierrez, J. M. Redwing, L. C. Lew Yan Voon, B. Lassen, and P. C. Eklund, Nano Lett. 4, 1663 (2004).

${ }^{17}$ R. S. Wagner and W. C. Ellis, Appl. Phys. Lett. 4, 89 (1964).

${ }^{18}$ G. Stibenz and G. Steinmeyer, Opt. Express 13, 2617 (2005). 